\section{Reduced vital capacity in ALS might be a cause of cognitive dysfunction}

Up to $50 \%$ of patients with amyotrophic lateral sclerosis (ALS) have cognitive impairment, most commonly fronto-temporal dysfunction. Some patients seem to have reversible dysfunction in addition to the irreversible degenerative process associated with the ALS. Studies have indicated that ventilatory status could impact on cognitive dysfunction in individuals with ALS.

Kim et al. measured cognitive functions related to the frontal and temporal lobes in 16 patients with early-stage ALS stratified by their vital capacity. Patients with reduced vital capacity (forced vital capacity $<80 \%$ predicted) performed significantly less well than those with a normal vital capacity ( $\geq 80 \%$ predicted) in tests of memory retention, retrieval efficacy, spoken verbal fluency and spoken verbal fluency index (which assessed time allocated for the task, time taken, and number of items generated). No such differences were evident when patients were grouped by disease severity rather than vital capacity.

The authors conclude that, in ALS, frontotemporal dysfunction might be partly the result of potentially reversible secondary effects associated with reduced vital capacity, such as hypoxia or sleep fragmentation. The study was not able to determine the mechanism by which reduced vital capacity affects cognitive function, but a large long-term prospective study is underway to further investigate the relationship between ventilatory status and cognitive function in ALS.

Original article Kim SM et al. (2007) Relation between cognitive dysfunction and reduced vital capacity in ALS. J Neurol Neurosurg Psychiatry [doi: 10.1136/jnnp.2006.111195]

\section{Can inhaled nitrous oxide prevent cerebral palsy in hypertensive newborns?}

Preterm infants with persistent pulmonary hypertension of the newborn (PPHN) are at high risk of serious neurodevelopmental complications resulting from hypoxemic respiratory failure. The benefits of treating hypoxemia with inhaled nitric oxide therapy (iNO) are uncertain, but the results of a recent historical study by
Tanaka et al. show that the risk of cerebral palsy in preterm infants with PPHN is lower in those treated with iNO than in those treated with $100 \%$ oxygen.

The study included long-term follow-up data for 31 preterm infants with hypoxemic respiratory failure resulting from PPHN who were treated with iNO $(n=16)$ or $100 \%$ oxygen $(n=15)$. After 3 years, the mortality rate did not differ between the treatment groups, but the incidence of cerebral palsy in patients treated with iNO was $12.5 \%$, compared with $46.7 \%$ in patients treated with $100 \%$ oxygen $(P=0.054)$. In multivariate logistic regression analyses adjusted for maternal fever during delivery, birth weight, Apgar score at $5 \mathrm{~min}$, high-frequency oscillatory ventilation, or surfactant therapy, iNO therapy was associated with a decreased risk of cerebral palsy compared with $100 \%$ oxygen therapy in preterm infants with PPHN.

These results are not consistent with those of earlier studies, but Tanaka et al. note that the risk reduction could be limited to preterm infants whose respiratory failure was caused by PPHN and previous studies were not limited to this population. These findings could have important implications for treatment, but further studies involving large numbers of patients are needed.

Original article Tanaka Y et al. (2007) Inhaled nitric oxide therapy decreases the risk of cerebral palsy in preterm infants with persistent pulmonary hypertension of the newborn. Pediatrics 119: 1159-1164

\section{PD confers an increased risk of essential tremor in relatives}

A study performed as part of the Mayo Clinic Family Study of Parkinson's Disease has reported that relatives of patients with Parkinson's disease (PD) have an increased risk of essential tremor (ET), suggesting that these conditions may share familial susceptibility factors. The findings were obtained through historical cohort analysis of a population-based sample of 981 first-degree relatives of 162 patients with $\mathrm{PD}$ and 838 first-degree relatives of 147 controls (individuals without PD, parkinsonism or tremor) from Olmsted County, MN, and analysis of a further 2,684 first-degree relatives of 411 sequential new patients with PD referred to the Mayo Clinic. 